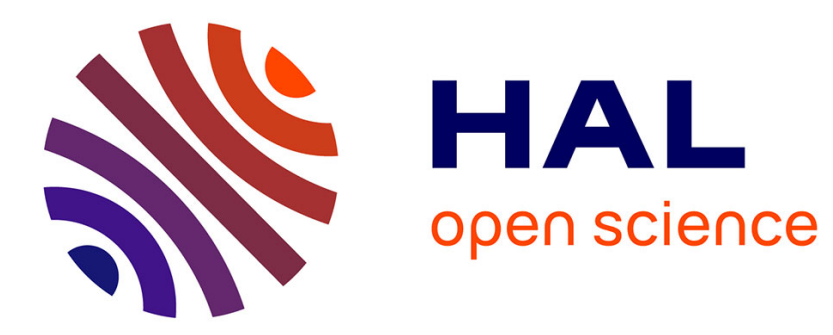

\title{
L'appropriation des terres agricoles, nouvelle étape de la mondialisation
}

Thierry Pouch

\section{To cite this version:}

Thierry Pouch. L'appropriation des terres agricoles, nouvelle étape de la mondialisation. L'Économie politique, 2018, 78 (2), pp.19-29. 10.3917/leco.078.0019 . hal-02050656

\section{HAL Id: hal-02050656 \\ https://hal.univ-reims.fr/hal-02050656}

Submitted on 8 Mar 2019

HAL is a multi-disciplinary open access archive for the deposit and dissemination of scientific research documents, whether they are published or not. The documents may come from teaching and research institutions in France or abroad, or from public or private research centers.
L'archive ouverte pluridisciplinaire HAL, est destinée au dépôt et à la diffusion de documents scientifiques de niveau recherche, publiés ou non, émanant des établissements d'enseignement et de recherche français ou étrangers, des laboratoires publics ou privés. 


\title{
L'appropriation des terres agricoles, nouvelle étape de la mondialisation
}

\author{
Thierry Pouch*
}

USQU'À TRĖS RÉCEMMENT, LA TERRE N'ÉTAIT PAS PRISE EN considération dans les analyses de la dynamique de transnationalisation des facteurs de production. La terre a en effet toujours été appréhendée comme un facteur non délocalisable. C'est pourquoi elle est régulièrement absente de la théorie des firmes multinationales. L'acquisition de terres par des allochtones est pourtant un processus historiquement ancien, à en juger par les colonisations successives qui ont notamment touché au XIXe siècle bon nombre de sociétés africaines.

Au début du XXIe siècle, la question foncière s'est installée dans le paysage mondial, avec ce que les médias et de nombreuses organisations non gouvernementales (ONG) ont régulièrement qualifié $d$ ' "accaparement des terres». Entre 2000 et 2006 , ce phénomène d'acquisition de terres reste encore marginal. Il faut attendre la crise de 2007 pour observer une croissance des investissements opérés par des capitaux étrangers dans des hectares de terres cultivables. Le foncier agricole est désormais de plus en plus convoité. Les achats ou locations d'hectares de terres cultivables ont depuis défrayé la chronique. Il suffit qu'un investisseur étranger - souvent chinois

* Thierry Pouch est économiste, chef du service études et prospective de l'Assemblée permanente des chambres d'agriculture (cet article n'engage pas cette institution), chercheur associé au laboratoire Regards de l'université Reims Champagne-Ardenne et membre de l'Académie d'agriculture de France. 
d'ailleurs - se porte acquéreur de plusieurs hectares cultivables pour susciter l'indignation des agriculteurs et la crainte de voir le pays dépossédé d'un outil de production, avec, en toile de fonds, une menace pesant sur la souveraineté alimentaire de la nation ou d'un groupe de pays. Au point que l'on a pu évoquer le risque d'un "néocolonialisme agraire », selon l'expression de l'ancien directeur général de l'Organisation des Nations unies pour l'alimentation et l'agriculture (FAO), Jacques Diouf.

La croissance des investissements dans le foncier agricole témoigne donc d'une mutation inédite de la mondialisation et

PAR LE TRUCHEMENT DES INVESTISSEMENTS

ÉTRANGERS, LA TERRE EST DEVENUE

UN FACTEUR DE PRODUCTION POUVANT FAIRE

L'OBJET D'UNE FORME DE DÉLOCALISATION.

ON ASSISTE À UNE " MARCHANDISATION » DU FONCIER interpelle les économistes sur plusieurs registres. Par le truchement de ces investissements étrangers, la terre est devenue un facteur de production pouvant faire l'objet d'une forme de délocalisation. On assiste à une « marchandisation » du foncier. Le premier registre sur lequel il est nécessaire de se pencher est celui de la genèse de ce processus d'accaparement des terres dans le monde. Le deuxième a trait à la mesure de ce phénomène économique, ainsi qu'à l'identification des pays cibles et des investisseurs. Peut-on dresser une typologie de ces acteurs et de leurs intentions ? Le troisième et dernier registre concerne l'interprétation économique que l'on peut faire des investissements dans le foncier cultivable. Le processus en cours ne fait que confirmer la généralisation des rapports marchands dans le monde, alors que la terre, comme la monnaie, peut être appréhendée par certains théoriciens hétérodoxes comme un bien collectif.

\section{Genèse de l'appétit de terres cultivables}

Par un curieux processus dont l'histoire économique a le secret, les matières premières agricoles ont effectué un saisissant retour à partir de la crise de 2007 . Le sentier de croissance sur lequel s'étaient précédemment engagées les éco-

[1] Une théorie

du capitalisme est-elle possible?, par Robert Boyer, Odile Jacob, 2004, ainsi que « La guerre économique, forme moderne de la guerre ", par Eric Bosserelle, Revue française de socio-économie, $\mathrm{n}^{0} 8$ pp. 167-186, 2011. nomies avancées, celui de l'immatériel, de la connaissance et de la finance, avait relégué au second plan la question des produits de base. La fin annoncée du capitalisme industriel était aussi celle des matières premières ${ }^{[1]}$.

La crise dite des subprimes a constitué une puissante force de rappel. D'abord, parce que l'explosion de la bulle 
immobilière a conduit les acteurs financiers à diversifier leurs placements et à investir dans des domaines plus sûrs et rentables. L'appétit pour les produits agricoles provient aussi de la tentative de résoudre l'équation pétrolière en produisant des énergies renouvelables issues de l'agriculture (agrocarburants comme l'éthanol). Enfin et surtout, les économies émergentes ont fini par émerger, par consolider leur puissance industrielle, bousculant la hiérarchie des nations. Ce processus s'accompagne d'un appétit de matières premières, entre autres agricoles, pour répondre aux besoins de populations qui s'accroissent et s'urbanisent toujours plus - rien qu'en Chine, le taux d'urbanisation a dépassé les $50 \%$ en 2016, les autorités ayant fixé comme objectif un taux de $70 \%$ à l'horizon 2050. Des populations dont le niveau de vie s'élève, occasionnant une transition nutritionnelle active. Ainsi, les régimes alimentaires s'occidentalisent en Asie.

Ces anciens pays émergents, qui tirent la croissance mondiale, sont toutefois pris en tenaille entre le nombre d'habitants à nourrir et le manque de surfaces cultivables, et ils s'exposent au risque de la dépendance alimentaire. La Chine, bien sûr, avec pas moins de $20 \%$ de la population mondiale pour seulement $8 \%$ de terres cultivables, mais aussi l'Indonésie, avec ses quelque 265 millions d'habitants (quatrième au classement de la population mondiale) qui ont vu leur niveau et leur mode de vie s'améliorer, les poussant à consommer viennoiseries, pâtisseries, pizzas et viandes en quantités plus importantes. Que dire des pays de la zone Afrique du Nord et Moyen-Orient, qui se distinguent par une forte sismicité alimentaire pouvant entraîner les sociétés dans un chaos politique et social, du fait des conditions climatiques et des répercussions à venir du réchauffement de l'atmosphère ? A l'horizon 2050, l'Algérie, par exemple, devrait voir sa surface cultivable se réduire de près de $50 \%$ sous l'effet du changement climatique, exacerbant une dépendance alimentaire déjà élevée ${ }^{[2]}$.

Quelles issues pour ces régions ? Par quels leviers sécuriser leurs approvisionnements alimentaires ? Le recours aux importations s'avère prioritaire. L'Indonésie et l'Egypte forment le duopole des importateurs mondiaux de blé, avec respectivement 13 et 12 millions de tonnes achetées durant la campagne 2017-2018. La Chine s’enfonce dans la dépendance alimentaire,
[2] Voir La dépendance alimentaire de l'Afrique du Nord et du Moyen-Orient à l'horizon 2050, par Chantal Le Mouël et Bertrand Schmitt (coord.), Quae, 2015. Ainsi que Géopolitique du blé. Un produit vital pour la sécurité mondiale, par Sébastien Abis, Armand Colin-Iris, 2015.

\section{\$>}


quasi absolue pour le soja, bien enclenchée pour les produits laitiers, les viandes bovine et porcine ainsi que, last but not least, pour les céréales, en dépit de sa position de premier producteur mondial de blé [3].

Importer comporte toutefois un risque, justement celui d'être placé sous la dépendance alimentaire d'un ou plusieurs pays fournisseurs, risque qui avait paradoxalement été bien pointé par David Ricardo, pourtant réputé comme l'un des, si ce n'est le plus grand

\section{SI IMPORTER REVIENT À S'EXPOSER AU RISQUE}

DE LA DÉPEN DANCE ALIMENTAIRE, INVESTIR DANS LE FONCIER AGRICOLE, REPRÉSENTE UNE ISSUE DÉSORMAIS EXPLOITÉE PAR DE NOMBREUX ACTEURS PUBLICS OU PRIVÉS des théoriciens du libre-échange. Dans son opuscule de 1815, Essai sur l'influence d'un bas prix du blé sur les profits, l'économiste britannique indiquait : «Seuls des arguments presque irréfutables

sur le danger de dépendre des pays étrangers pour une partie de notre nourriture, car c'est seulement dans cette optique qu'il peut y avoir discussion, devraient être avancés pour nous amener à restreindre les importations. Ce qui nous obligera aussi à retenir du capital dans un emploi qu'il quitterait sinon pour un autre beaucoup plus rentable. [...] Si de mauvaises saisons survenaient outre-mer, les pays exportateurs seraient en droit de nous refuser la quantité habituellement exportée, pour pallier leur propre déficit » ${ }^{[4]}$.

Si importer revient à s'exposer au risque de la dépendance alimentaire, investir dans le foncier agricole, dans des hectares de terres cultivables disponibles de par le monde, représente une issue désormais exploitée par de nombreux acteurs publics ou privés. C'est pourquoi l'on assiste depuis le milieu des années 2000 à une envolée des investissements dans le foncier. La vague actuelle d'acquisitions foncières n'est certes pas la première dans l'histoire du monde. Certains auteurs ont en effet montré que, du fait des colonisations et du développement de cultures d'exportation au cours du XIXe siècle, il y eut une première phase d'investissements étrangers dans la production agricole ${ }^{[5]}$.

Enfin, des déterminants plus locaux peuvent être à l'origine de transactions sur le marché du foncier agricole. Par exemple, les agriculteurs blancs d'Afrique du Sud, confrontés au déploiement d'une réforme agraire visant à redistribuer les terres, ont 
pu, au début des années 2000, louer 200000 hectares au Congo Brazzaville en accord avec le gouvernement local.

L'une des questions essentielles de la littérature économique actuelle traitant de l'accaparement des terres concerne sa définition et sa mesure. Les premières tentatives se sont souvent soldées par une approche trop simplifiée du processus, sans doute parce qu'elles émanaient de la sphère médiatique ou des ONG. Etaient retenus les projets d'acquisitions foncières à grande échelle, portés par des Etats du golfe arabo-persique ou la Chine, sans véritablement discerner les ambitions des investisseurs (agriculture, foresterie, voire tourisme...). Plusieurs observateurs ont a contrario plaidé pour un recentrage de l'analyse sur des investissements destinés à des finalités agricoles, assorti d'une identification précise et variée des acteurs (privés, publics, nationaux et internationaux) ${ }^{[6]}$. De plus, cette difficulté à définir l'acquisition d'hectares de terres exerçait une lourde contrainte sur la mesure du phénomène, du fait de l'incohérence des données, voire de leur absence.

Plusieurs chercheurs sont toutefois parvenus à proposer une définition rigoureuse de l'accaparement/acquisition de terres. Selon l'équipe réunie autour de l'économiste Ward Anseeuw, une acquisition foncière se traduit par " un transfert de l'usage, du contrôle ou de la propriété de la terre via la vente, le prêt
[6] « Contextualising Land Grabbing :

Contemporary Land Deals, the Global Subsistence Crisis and the World System ", Canadian Journal of Development Studies, 33 (20), pp. 119-142, par A. Haroon Alkam-Lodhi, 2012.

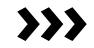

Acquisitions foncières internationales cumulées de 2000 à 2016

\begin{tabular}{|l|c|c|c|}
\hline & Nombre de cas & $\begin{array}{c}\text { Surfaces demandées, } \\
\text { en millions d'hectares }\end{array}$ & $\begin{array}{c}\text { Surfaces transférées } \\
\text { en millions d'hectares }\end{array}$ \\
\hline Accords verbaux & 72 & 4,6 & 2,2 \\
Contrats signés & 1132 & 52,6 & 40,3 \\
Projets conclus & 1204 & 57,2 & 42,5 \\
\hline Expressions d'intérêt & 57 & 8,3 & $\mathrm{~s} / \mathrm{0}$ \\
Négociations en cours & 155 & 12 & $\mathrm{~s} / \mathrm{0}$ \\
Projets annoncés & 212 & 20,3 & $\mathrm{~s} / \mathrm{O}$ \\
\hline Echecs & 63 & 6 & $\mathrm{~s} / \mathrm{0}$ \\
des négociations & & & 0,9 \\
Contrats annulés & 34 & 1,2 & 0,9 \\
Echecs des projets & 97 & 7,2 & \\
\hline
\end{tabular}

Source : Land Matrix, 2016 (s/o : sans objet) 
ou la concession d'une surface supérieure à 200 hectares à un investisseur étranger $»[7]$.

\section{Mesurer les investissements dans le foncier agricole}

Quel vocable choisir pour qualifier au mieux le processus qui s'est abattu sur le foncier agricole ? Malgré son utilisation répandue, le terme anglais "land grabbing » paraît inadapté parce qu'il évoque une action violente pour déposséder autrui de son outil de production, tout en mésestimant les conséquences de la concentration d'hectares entre les mains de l'acquéreur ${ }^{[8]}$. «Appropriation de terres » est manifestement l'expression qui convient le mieux pour décrire la dynamique foncière à l'échelle mondiale : elle ne contient pas l'idée d'un usage de la force et reflète au contraire celle d'une transaction commerciale avec

[7] Transnational Land Deals for Agriculture in the Global South. Analytical Report Based on the Land Matrix Database, CDE-Cirad-Giga par Ward Anseeuw et al., 2012.

[8] « Les accaparements de terres dans le monde une menace pour tous ", par Michel Merlet, Grep/ Pour $\mathrm{n}^{\circ}$ 220, 2013 pp. 95-104.

[9] Centre de coopération internationale en recherche agronomique pour le développement : German Institute for Global and Area Studies Deutsche Gesellschaft für Internationale Zusammenarbeit ; Center for Development and Environment ; International Land Coalition ; landmatrix.org.

[10] Land Matrix offre donc une source d'informations comblant un vide, en dépit des limites de cet observatoire mondial : petites surfaces non répertoriées concentration des hectares entre les mains de quelques gros investisseurs, peu, voire pas d'analyses des conséquences en matière de systèmes agraires... transfert partiel ou total de propriété au profit d'un acteur étranger pouvant conduire à des formes de concentration des terres - ce qui bien évidemment n'exclut pas que, dans la négociation, des pressions puissent s'exercer pour convaincre le pays hôte de céder quelques centaines ou milliers d'hectares.

Il existe aujourd'hui une base de données exploitable, créée en 2000 - Land Matrix -, pour mesurer l'ampleur des investissements réalisés dans le foncier agricole et en livrer une image plus précise. Ces données sont recensées et classées par cinq institutions : en France, le Cirad, en Allemagne, le Giga et le GIZ, en Suisse le CDE et l'ILC [9]. Les ambitions des investisseurs pour des surfaces de terres agricoles ne se traduisent pas toujours par un transfert réel de propriété. Bon nombre de projets sont abandonnés en cours de route, la négociation entre les parties cocontractantes n'aboutissant pas. La base de données Land Matrix offre de ce point de vue l'opportunité de clarifier ce qui relève de l'intention, du transfert de propriété (achat) ou encore de la location avec des baux emphytéotiques. Le recensement auquel procède Land Matrix recouvre les transactions réalisées depuis 2000 pour des surfaces égales ou supérieures à 200 hectares, qui occasionnent une mutation de l'usage des terres et débouchant sur des productions agricoles commercialisées ensuite sur le marché ${ }^{[10]}$.

Le tableau page précédente fournit un panorama, en cumulé sur 2000-2016, des opérations foncières selon le statut de la négociation. On remarque ainsi que, sur la période, ce sont plus de 42 millions d'hectares de terres qui ont été appropriés par des 
allochtones. Sur ce total, 24 millions d'hectares correspondent à des usages agricoles, 12 millions à l'exploitation forestière et le reste à d'autres usages (tourisme, industries, etc.).

Cette photographie annuelle des acquisitions de terres dans le monde doit toutefois être remise dans une perspective historique. Un tel exercice indique un accroissement fulgurant des investissements depuis le début des années 2000, mais la période récente laisse entrevoir un plafonnement du processus. Celui-ci s'explique non seulement par la variété des investissements possibles à réaliser pour les détenteurs de capitaux (industrie, finance, etc., en fonction des différentiels de rentabilités), par les échecs des acquisitions foncières en termes de projets productifs, par

SUR LA PÉRIODE 2000-2016, CE SONT PLUS DE 42 MILLIONS D'HECTARES DE TERRES QUI ONT ÉTÉ APPROPRIÉS PAR DES ALLOCHTONES. SUR CE TOTAL, 24 MILLIONS D'HECTARES CORRESPONDENT À DES USAGES AGRICOLES

la baisse des prix des produits agricoles depuis 2014, mais aussi par la prise de conscience par les pays qui cèdent leurs terres de la fragilité de leur souveraineté alimentaire, leur suggérant d'adopter des législations limitant l'implantation d'investisseurs étrangers sur leur sol.

L'impact des mouvements paysans de contestation a exercé un effet notoire, comme en témoigne le cas devenu emblématique des agriculteurs malgaches : leur action a fait échouer les appétits du géant industriel sud-coréen Daewoo, a précipité la chute du gouvernement de Marc Ravalomanana et l'accession au pouvoir en 2009 d'Andry Rajoelina. Ce groupe coréen avait négocié l'acquisition en 2008 d'1,3 million d'hectares sans coût, avec pour contrepartie des investissements dans des infrastructures portuaires à hauteur de 6 milliards de dollars sur un quart de siècle, qui devaient déboucher sur la création de 70000 emplois. Outre que cette opération a été perçue comme une menace pour la souveraineté du pays, les paysans se sont sentis spoliés de leur outil de travail. Sans parler du fait que la terre est le lieu où reposent les ancêtres, ajoutant ainsi une dimension symbolique à la contestation sociale. L'expérience malgache indique que le climat social dans les pays ciblés par les investisseurs sera à l'avenir l'un des freins possibles à la convoitise dont la terre fait l'objet.

Cette approche globale mérite d'être complétée par une typologie des investisseurs et des pays cibles. Les données 
fournies par Land Matrix permettent de dresser une typologie des 20 principaux pays investisseurs. Loin des commentaires simplistes et hâtifs entendus notamment dans les médias, qui ont souvent stigmatisé des acteurs chinois, il est assez remarquable de constater que la Chine n'arrive qu'au neuvième rang, avec 1 million d'hectares acquis. Quant à la "Chine élargie », incluant Hongkong, elle se situe au troisième rang. La Malaisie est le premier investisseur mondial dans le foncier agricole avec plus de 3,5 millions d'hectares, suivie par les Etats-Unis (plus de 3 millions d'hectares). Viennent ensuite le RoyaumeUni (près de 2 millions d'hectares), puis Singapour $(1,6)$, l'Arabie Saoudite $(1,4)$, l'Inde $(1,3)$, les Pays-Bas $(1,2)$ et, plus loin derrière, la France, le Canada, le Luxembourg et la Corée

\section{LES APPROPRIATIONS D'HECTARES DE TERRES}

ONT MAJORITAIREMIENT LIEU EN AFRIQUE

SUBSAHARIENNE. LA VARIÉTÉ DES RÉGIMES

FONCIERS PERMET AUX INVESTISSEURS

D'Y ACQUÉRIR DES SURFACES EN JOUANT

SUR LA COMPLEXITÉ DES STATUTS JURIDIQUES du Sud. Ce dernier pays a axé sa stratégie agricole sur la monoculture du riz. Ne parvenant pas à équilibrer ses approvisionnements alimentaires, hors riz, par des productions domestiques, la Corée du Sud est devenue au début des années 2000 un acteur important sur le marché mondial des terres agricoles, figurant parmi les 20 premiers investisseurs mondiaux. Elle a, en quelque sorte, inversé le processus dont elle fut elle-même victime il y a un siècle face à un Japon alors en recherche d'hectares de terres cultivables ${ }^{[11]}$. Dans leur grande majorité, les investisseurs sont d'abord des entreprises cotées en Bourse (18\%), d'autres entreprises privées ( $16 \%$ ) et des fonds d'investissements (15\%). Viennent ensuite des Etats à hauteur de $20 \%$, puis des entrepreneurs individuels pour $7 \%$.

Cette typologie en appelle une seconde, ayant trait aux finalités des acquéreurs. Là encore, Land Matrix livre des informations chiffrées intéressantes qui dessinent un paysage contrasté. Les transactions réalisées avec les parties prenantes locales, en particulier les Etats, sont majoritairement destinées aux flex crops, cultures dont la production peut être orientée vers les agrocarburants ou vers des usages alimentaires (canne à sucre, maïs, soja et huile de palme). En 2016, la production d'agrocarburants représentait $34 \%$ des projets d'investissements, suivie par les cultures alimentaires pour $13 \%$. En conséquence, les acquisitions de terres peuvent déboucher sur des modèles de plantations à grande 
échelle, à l'instar de celles de la Malaisie qui, contrainte par une insuffisance de surfaces disponibles sur son sol, a investi depuis quelques années en Indonésie pour étendre sa production d'huile de palme.

Une troisième typologie a trait aux pays cibles. Il ressort de l'exploitation des données de Land Matrix que le continent africain est celui sur lequel le nombre de transactions réalisées est le plus élevé (422 entre 2000 et 2016 , pour plus de 10 millions d'hectares). L'Asie se classe deuxième, avec 305 contrats pour près de 5 millions d'hectares, devant l'Amérique latine (146 contrats pour 4,5 millions d'hectares), l'Europe de l'Est (96 pour 5,1 ) et l'Océanie (35 pour 2,2). Les 20 premiers pays cibles représentent près de 22 millions d'hectares, soit $82 \%$ des transactions conclues sur les cinq continents. L'Indonésie est la première destination mondiale des investisseurs, suivie de l'Ukraine et de la Russie, de la Papouasie-Nouvelle Guinée et du Brésil. Quant à la France, concernée par les convoitises de quelques investisseurs, en particulier chinois, elle ne figure pas dans le classement des 20 principales destinations. Rappelons toutefois que $3 \%$ du vignoble bordelais appartiennent désormais aux Chinois et que 1700 hectares de terres agricoles céréalières ont été achetés en 2016 dans l'Indre par un fonds de gestion chinois basé à Hongkong qui en a également acquis 900 dans l'Allier en 2017.

Les appropriations d'hectares de terres ont majoritairement lieu en Afrique subsaharienne. La variété des régimes fonciers (domanialité publique, propriété privée, possession coutumière...) permet aux investisseurs d'y acquérir des surfaces en jouant sur la complexité des statuts juridiques et en profitant de politiques publiques peu protectrices des intérêts des paysanneries locales. La dynamique d'appropriation des hectares de terres est en effet sous-tendue par la question des régimes fonciers. C'est sur ce point que se concentrent le plus souvent les critiques des ONG, qui dénoncent la spoliation des paysans locaux. En revanche, de nombreuses institutions internationales, Banque mondiale en tête, prônent l'extension de la privatisation des terres ${ }^{[12]}$. Elles relaient en cela la position des investisseurs qui estiment que leurs capitaux ont pour finalité la mise en valeur de terres jusque-là peu, voire pas du tout exploitées. Ce qui aboutit à une meilleure allocation du capital foncier ou, dans certains cas, à une
[12] Il s'agit d'une dimension normative en économie. Lire notamment Armen A Alchian et Harold Demsetz, "The Property Right Paradigm ", The Journal of Economic History, vol. 33 , $n^{0} 1,1973$, pp. 16-27. Voir également Benoît Lallau, " Land Grabbing versus investissements fonciers à grande échelle. Vers un "accaparement responsable" ? ", L'Homme et la Société, nos 183-184, 2012, pp. 15-34.

\section{\$\$}


valorisation d'espaces forestiers pour des productions agricoles. Mais les répercussions humaines peuvent être importantes, à l'image de celles subies par les Pygmées, victimes de la conversion de forêts en surfaces dont sont issues des productions agro-industrielles tournées vers l'exportation. C'est pourquoi la vague d'investissements directs dans le foncier attise parfois les rapports de force et bloque les réformes agraires visant à faciliter l'accès à la terre pour des paysans qui en sont dépourvus.

\section{Une marchandise nommée terre}

Le déploiement d'investissements dans des hectares de terres situés à l'étranger s'inscrit dans un contexte de mutation des conditions de l'accumulation du capital dans le capitalisme globalisé. Le coût en capital de la production de biens alimentaires s'est accru ces dernières années. Les grandes puissances émergentes se situent sur une telle trajectoire. Ce qui signifie que l'appropriation de surfaces agricoles a pour finalité de produire sur un sol étranger des biens alimentaires capables de répondre aux besoins de la population. De plus, le développement des productions d'énergies renouvelables de type éthanol hypothèque depuis le début de la décennie 2000 les disponibilités agricoles à usages alimentaires, incitant les pays en manque de terres, en situation de quasi-dépendance alimentaire et de précarité énergétique, à investir dans le foncier agricole à l'étranger pour accéder directement aux productions.

Une telle tendance coïncide avec la dynamique de libéralisation des échanges de produits agricoles et alimentaires, initiée par les accords de Marrakech signés en 1994. Après la phase de démantèlement des politiques agricoles, tant dans les pays industrialisés que ceux en développement, l'ouverture commerciale qui en a résulté était porteuse d'une seconde phase, celle des acquisitions de terres. Cette dernière s'articule avec la financiarisation du capitalisme, laquelle a facilité, en décloisonnant les marchés, les flux de capitaux d'un secteur à un autre. Les acquisitions d'hectares de terres forment ainsi un nouvel espace de conquête. Marchandisée, la terre offre désormais au capital une opportunité de se métamorphoser en investissant soit dans une perspective de pure spéculation, soit pour en retirer une rente. 
La crainte de voir resurgir des flambées de prix agricoles, comme durant les années de crise des subprimes, les incertitudes relatives aux ressources énergétiques non renouvelables comme le pétrole, les contraintes écologiques pesant sur les productions dans les pays industrialisés (plus légères dans les pays du Sud) sont autant d'équations qui trouvent leur mode de résolution dans les appropriations d'hectares de terres, pour former ce que les théoriciens des régimes alimentaires ont nommé une "nouvelle bioéconomie » ${ }^{[13]}$.

En acquérant des hectares de terres cultivables, majoritairement

\section{EN ACQUÉRANT DES HECTARES DE TERRES} CULTIVABLES, LES INVESTISSEURS EN FONT UN OBJET DE TRANSACTION, UNE CHOSE INERTE, ÉCHAPPANT AUX RELATIONS SOCIALES QUI FURENT LE SUBSTRAT D'IDENTITÉS, D’ÉQUILIBRES, DE CROYANCES... dans les pays de l'hémisphère Sud, les investisseurs font de la marchandise échangée - la terre un objet de transaction, une chose inerte, échappant ipso facto aux relations sociales qui, jadis, furent le substrat d'identités, d'équilibres, de croyances... La terre est en quelque sorte désocialisée ou, pour l'exprimer à la façon de Karl Polanyi, "désencastrée ». Elle n'est plus le pivot du système social. Ces appropriations de terres constituent des formes modernes d'expropriation des paysans, à l'image du mouvement des enclosures tant décrites par Karl Marx dans le chapitre du Capital dédié à "l'accumulation primitive du capital ». Le processus actuel d'acquisition de terres met en quelque sorte au jour une recomposition des rapports sociaux de production et une extension des formes de l'accumulation primitive du capital à l'échelle mondiale [14].

Les convoitises des puissances capitalistes sur les terres cultivables corroborent le fait que la coupure radicale enclenchée à la fin du XVIII e siècle entre l'économie et le tissu social semble se poursuivre sans relâche. Elles illustrent également les stratégies déployées par les grands acteurs capitalistes pour contrôler les ressources naturelles. Au XIXe siècle, la colonisation formait l'une des voies possibles du contrôle des ressources. Aujourd'hui, il s'agirait plutôt de détenir la propriété et les conditions d'usage du sol sans accaparer les structures de l'Etat. Les relations internationales sont bel et bien un espace de rivalités entre des puissances qui s'engagent de plus en plus dans une lutte pour capter les ressources et pour le partage des marchés ${ }^{[15]}$.

[13] Lire notamment Philip McMichael, « The Land Grab and Corporate Food Regime Restructuring ", Journal of Peasant Studies, 39 (3-4), 2012, pp. 681-701.

\footnotetext{
[14] Cf. Karl Polanyi (1944), The Great Transformation, New York, traduction française Gallimard, 1983, ainsi que Jacques Le Goff, La civilisation de l'Occident médiéval, Arthaud, 1964, et Louis Dumont, Homo Aequalis. Genèse et épanouissement de l'idéologie économique, Gallimard, 1985.
}

[15] Sur cette vision des relations internationales on peut se reporter à Vladimir Ilitch Lénine (1916), L'impérialisme, stade suprême du capitalisme, Science marxiste, 2005, pour la nouvelle édition française. 